respond to an inoculation in the same degree as a patient with a low index.

(1) Antitryptic index $=+0 \cdot 5$. Index of serum after inoculation to that before $=+0.07-$

(2) Antitryptic index $=+0.4$. Index of serum after inoculation to that before $=+0.1-$

(3) Antitryptic index $=+0.08$. Index of serum after inoculation to that before $=+0 \cdot 2$

(4) Antitryptic index $=$ Normal. Index of serum after fnoculation to that before $=+0.22$

In each case the inoculation consisted in the administration of 0.001 gramme of tuberculin.

CASE 2.-A young man, aged 23 years, discharged from the army with well-marked tuberculous infiltration of both lungs. This patient, who was under the care of Mr. Hort, reacted well to treatment and after four months was discharged with the disease arrested. Throughout the first two months of treatment he had an irregular temperature, ranging from $99^{\circ}$ to $101^{\circ}$; during the third month the temperature gradually became normal. On admission the index was on the minus side, being -0.01 . Two weeks later it was found to be normal and remained normal till the time of the patient's discharge. It may be noted that throughout the whole course of his treatment he steadily gained in weight. The reaction to inoculation by 0.001 tuberculin was taken on five occasions. On admission the serum after inoculation, as compared with that before, showed a rise of index of +0.5 . The patient was inoculated twice a week for five weeks, at the end of which period an index of +0.06 was obtained. At the end of the third month the postinoculative index was +0.08 . Two further observations gave no rise of index at all towards the end of the patient's treatment.

CASE 3.-A woman suffering from tuberculous involvement of the whole of the left lower lobe gave at the beginning of her treatment an antitryptic index of $+0 \cdot 18$. Examined three months later, when the disease appeared to have been arrested, the incex was normal. In this case no inoculations were performed but the patient had been treated by the method of auto-inoculation by Mr. Hort. Two other cases undergoing cure showed a variation of antitryptic activity in the same direction.

Two cases dying from acute pulmonary tuberculosis were examined shortly before death; in both cases the antitryptic index was high, being +0.4 and +0.5 respectively. In a series of 33 inoculations studied in tuberculous patients a rise of antitryptic index followed the inoculation in 24 cases, and the amount of rise appears to vary inversely to the antitryptic index in the patient as regards normal. The method would appear to afford additional evidence, were any needed, of the efficacy of the oral administration of tuberculous vaccines introduced by $\mathrm{Dr}$. Latham. In four cases in which inoculation was practised on non-tuberculous subjects no rise was noted. Attempts have been made to find the time relations of the post-inoculative rise. The earliest period at which the rise was found was four hours after a subcutaneous inoculation. The index appears to return to its former level in about 40 hours if the dose be not so excessive as to cause marked reaction. In no case was a negative phase noted. Such rises of index after inoculation appear to occur independently of a rise of tem. perature, but in those cases in which no rise was noted there was never any increase of temperature succeeding inoculation.

The method of giving serum by the mouth together with vaccine has been used by Dr. Latham and he has noted that the vaccine can be given in this method in much larger doses. In a case under his care it was found that no rise of index followed an inoculation of ${ }_{30}^{1} \delta$ of tuberculin and on inquiry it was found that the dose had been given with five cubic centimetres of normal horse serum. After a few days the dose was repeated with the serum omitted and on this occasion a rise of index of +4 was noted. In three cases of tuberculous disease of the joints no rise of the antitryptic index was found and results from inoculation proved to be negative. In a case of tuberculous glands operation was followed by a rise of antitryptic index of $+0 \cdot 2$ on the morning after operation.

It is hoped that if further researches confirm and extend the conclusions presented in this preliminary note we may be in possession of an easy physical method which has a negligible experimental error and unlike opsonic determinations does not necessarily remain in the hands of a few highly-skilled observers. By such a method the dosage of inoculation may be surely gauged when the temperature chart affords no help as occurs so frequently in cases of febrile tuberculosis. The index may also prove of prognostic value and the results hitherto arrived at seem to show that it is possible by its means to decide what cases are in need of inoculative treatment.

The materials so far at our disposal do not permit the discussion of the significance of the variations of the antitryptic power of the blood. Whether it be an expression of the attempt on the part of the organism to protect damaged cell tissue from autolysis, as Baer has demonstrated in vitro when serum is added to autolysing tissue, or whether it be an expression of the changes in antitryptic content evoked by the inoculation of the organism with the tryptic ferments from damaged and disintegrating leucocytes, are questions which must be determined by other methods than those of clinical observation. At present work is being carried on to determine the variation that may occur in the antipeptic power of the blood. As Opie and others have shown, both tryptic and peptic ferments occur in leucocytic ferments, and the latter occurs in the lymphocytes alone, whilst in the polymorphonuclear cells the tryptic ferment is predominant. Attempts are also being made to determine the question of the specificity of serum antiferments to the endo-enzymes of different tissues. A line of inquiry that much requires elucidation is one to determine the question as to whether variations of antitryptic power are due to increase or diminution of the antitryptic part of the serum or to variations in amount of the auxylytic substance which is generally supposed to be attached to the globulin moiety. In conclusion, I wish to express my thanks to Dr. Waller, in whose laboratory this work has been conducted, for his kindness in affording me every facility for my experiments.

Bibliography.-Miiller and Jochmann : Münchener Medicinische Wochenschrift, 1906, No. 4. For references to further literature see Miiller, Deutsches Arehiv für Klinische Medicin, 1907, Band 91-3. Bittorf: Ibid., vol. xci., No. 1-2. Wiens: Ibid., vol. xci,, No. 5-6 Münchener Medicinische Wochenschrift, 1907, No. 53. Brieger and Trebing : Berliner Klinische Wochenschrift, 1908, No. 22. Bergmann and Meyer: Ibid., 1908, No. 37. Bayliss: Archives des Sciences Biologiques, 1904, p. 261.

\section{SPONTANEOUS CURE OF THORACIC ANEURYSM.}

\section{By SIR THOMAS OLIVER, M.D. GLASG., LL.D.,} D.SC. SHEFF., F.R.C.P. LoND.,

PROFESSOR OF PHYSIOLOGY IN THE COLLEGE OF MEDICINE, AND PHYSICIAN TO THE ROYAI VICTORIA INFIRMARY, NEWCASTLE-UPON-TYNE.

LATENT thoracic aneurysm unattended by symptoms and physical signs, yet suddenly rupturing and causing immediate death, is not unknown, but with the spontaneous cure of thoracic aneurysm many of us are not familiar. As the possibility of such an occurrence is even questioned by some physicians I may be excused for bringing the following case under the notice of the readers of THE LANCET. The case was that of a man who, although the subject of recurrent dyspnoea especially of a nocturnal character, presented on examination no physical signs of disease of the aorta. In him the difficulty of breathing took the type of renal asthma and was relieved by the treatment usually adopted for it. At this stage in the history of the case and with all the details of the necropsy before me there seems to be nothing to alter in regard to what has been stated as to the cause of the shortness of breath.

The patient, a man aged 55 years, was admitted into the Royal Victoria Infirmary, Newcastle-upon-Tyne, on April 15th, 1908, suffering from extreme weakness and difficulty of breathing. Seven years ago he was an in-patient of the Royal Infirmary, Liverpool, on account of acute rheumatism from which he had suffered for four years previously. 12 years ago, while employed in marine works in Philadelphia, U.S.A., he lost his wife and four children all within 48 hours from malarial fever, the disease being epidemic in the city at the time. Four years ago the patient was suddenly seized with vomiting and diarrhœa. The 
attack only lasted two days but in the act'of romiting and retching he felt "something suddenly give way in the chest. Shortly afterwards pain was felt in the left chest and his breathing became difficult." In consequence of this he gave up his appointment in America and coming to London he became an in-patient of Charing Cross Hospital. With rest and treatment he recovered sufficiently to be able to take up work again, and he continued working for a period of 15 months. At the end of this period as "he was not feeling well he took a rest for six weeks, when he was again able to resume his duties which he discharged for six months.

When the patient came under my care he was the subject of difficulty of breathing of an intermittent character which was always worst at night. The arterial tension as estimated by the Riva-Rocci apparatus was equal to 180 millimetres of mercury. The radial pulse, which was rapid and irregular, was equal on both sides. Cardiac dulness commenced in the line of the left nipple and was carried to the right border of the sternum. Over the base of the heart the second sound was accentuated, while over the apex area the first sound was murmurish, feeble, and often indistinct. No apex beat could be felt. Owing to the difficulty of breathing the patient, especially at night, frequently occupied the semiupright position. Beyond the signs of slight pulmonary emphysema and the presence of a certain amount of dulness over the base of the left chest posteriorly and in the axillary region nothing abnormal was detected on percussion. The respiratory murmur was coarse over the upper part of each lung, while over each base crepitation was heard. The urine had a specific gravity of 1025 and 22 ounces were passed daily; it contained a large quantity of albumin. The abdomen presented nothing abnormal. There was no codema of the feet. The attacks of difficulty of breathing were relieved by inhalations of nitrite of amyl.

Three weeks after his admission into the infirmary the patient began to spit up blood apparently from the left lung. The breathing became Cheyne-Stokes in character, the periods of apnca lasting frequently from 40 to 60 seconds. The urine fell to six ounces daily and contained blood. Five grains of theocine sodium acetate were administered every three hours and within 12 hours the urine-rose to 84 ounces and on the following day to 96 ounces. For several nights the patient slept better; he seemed much improved and the bleeding from the lungs and the kidneys entirely ceased. The improvement only lasted a short while, for the patient was suddenly seized with acute pain over the right side of the chest. Over the affected area friction could be heard. The dulness over the base of the left lung increased and the respiratory murmur became almost inaudible. By degrees the quantity of urine diminished, the breathing became again difficult, and the pulse and the heart sounds feebler. The patient died exactly one month after his admission into the infirmary.

For the following notes of the post-mortem examination I am indebted to Dr. George Hall, pathologist to the Royal

Victoria Infirmary :-

There is no cedema of the extremities. The pericardium contains a small quantity of free fluid. The left pleural cavity contains about two pints of fluid, in consequence of which the lower lobe of the lung is compressed. The right chest shows signs of recent pleurisy. In addition to the collapse just mentioned the lungs are cedematous at the bases and at their margins are emphysematous. The heart is large. The parietal pericardium over the left ventricle is thickened. The mitral orifice admits the tips of two fingers; its cusps are normal. The cavity of the left ventricle is slightly dilated and its walls considerably thickened. The aorta at its oxigin showed several fatty and atheromatous patches, between matous patches, between which are interspersed ealcareous plates. there is a well-defined aneurysmal dilatation of the vessel which extends almost to the origin of the left carotid and subclavian arteries while beye origin of the left carotid and me the commencement of the descending aorta a fusiform dilatation of the vessel. The main aneurysm which is saccular has a definite daughter saccule nearly the size of a golf ball springing from its anterior surface and pointing forwards. The dilatation of the aorta extends to the root of the innominate artery but not into the vessels arising from it. The interior of the aneurysm is occupied by firm decolourised clot but this does not extend into the innominate artery to block it. At places the clot is fimly of the aneurysin falls to detect any involvement of nerves in the sac. The tricus or The pulmonary artery and valves are healthy. Liver and spleen call for tho con a cyst of the size of a chestnut, the capsule being stripped off leaves a rough surace and se left kidney shows similar changes to those the substance tough. The loft kn observed in the right. Both may be described as pale contracting kidneys.
Whatever may be our opinion in regard to the gravity of thoracic aneurysm it is within the experience of physicians that patients who are the subjects of this affection may not only live a considerable time after their malady has been detected but that even alarming and distressing symptoms may temporarily subside or even entirely disappear. Death does not always come by rupture of the sac, threatening at times as this may seem. Where improvement has taken place for a period and the patient has subsequently succumbed to an intercurrent malady, the aneurysm at the necropsy is frequently found to have been undergoing a process of cure, nature's method of accomplishing this being the strengthening of the walls of the sac by the internal deposition of layer after layer of clot which diminishes. the shock imparted to the sac by each systole of the left ventricle. While such a deposition of fibrin oecurs in aneurysms situated at some distance from the heart and occasionally leads to spontaneous cure it is maintained by some patbologists that the conditions of quietude, lowered arterial tension, and slowing of the circulation, which are essential to the formation of clot within an aneurysmal sac are almost impossible when the aneurysm is near the heart; for example, on the ascending portion of the arch of the aorta. Notwithstanding all these it is yet a fact that in a large number of cases of thoracic aneurysm, especially of the sacculated type, clot is found in the interior, indicating an effort on the part of nature to establish a cure.

The presence or absence of coexisting aortic regurgitation is not without importance in regard to the deposition of fibrin in thoracic aneurysm. If there is aortic incompetence of any severity there can hardly be in the interior of a thoracic aneurysmal sac the same favourable conditions for clotting likely to appertain where there is no aortic reflux, for in uncomplicated cases of thoracic aneurysm. the left ventricle of the heart is usually not hypertrophied to any extent, if at all, while it is enlarged in aortic regurgitation. The fact that patients occasionally live for several years after their thoracic aneurysm has" been detected can only be explained by the presence of the firm clot in the interior of the sac observed after death.

A few of my own patients suffering from thoracic: aneurysm lived seven or eight years, and in one case even. longer, after first coming under observation, others for several months, notwithstanding recurrent and copious: hæmorrhages. As bearing upon this question of longevity of patients suffering from thoracic aneurysm and their not being disqualified for work which necessitated considerable strain, I cannot do better than quote the case of Dr. George A. Gibson of Edinburgh alluded to by the late Sir William T. Gairdner in Allbutt's System of Medicine. ${ }^{1}$ The patient was a man, aged 39 years, a lamp-lighter by occupation, who lived for 12 years after his aneurysm was detected and who during that period frequently "undertook the duties of the keeper of one of the Edinburgh monuments which involved his ascending sometimes 50 times a day to a height of almost. 200 feet."

Although it is by the deposition of clot within the sac that the further development of aneurysm is checked and the physical signs of the tumour as well as the symptoms caused by it become altered in character there are, it need hardly be mentioned, different kinds of clot to be considered in this relationship. Cure can only take place through organisation of the clot. This occurred so completely in the patient. whose case is the subject of these notes that although a large aneurysm of the thoracic aorta was found after death it was consolidated and its clot so thoroughly organised that it gave rise to no symptoms, it had apparently ceased to exist. and in no way contributed to the fatal termination. On microscopical examination of sections of the aneurysm the outer part of the clot is observed to have become transformed into dense layers of fibrous tissue. Organisation has completely taken place. Numerous large spindleshaped fibroblasts can be seen in the outer half of the thrombus. The altered layers of fibrin are observed to be becoming encroached upon by new capillaries and granula tion tissue cells

Newcastle-upon-Tyne. 